\title{
Characterization of the maintained vegetative phase deletions from diploid wheat and their effect on VRN2 and FT transcript levels
}

\author{
Assaf Distelfeld · Jorge Dubcovsky
}

Received: 19 October 2009 / Accepted: 30 December 2009 / Published online: 9 January 2010

(C) The Author(s) 2010. This article is published with open access at Springerlink.com

\begin{abstract}
Allelic differences at the VRN1 (API/CAL/ $F R U), V R N 2$ (ZCCT) and VRN3 (FT) vernalization genes affect flowering time in wheat. The two maintained vegetative phase (mvp) mutants from Triticum monococcum L., previously reported as carrying a single gene (VRNI) deletion, are incapable of flowering. In this study, we show that both $m v p$ lines have larger deletions that include the genes AGLG1, CYS, PHYC, VRN1 and possibly others. The original $m v p$ deletions were generated in lines that lack the $V R N 2$ gene. Therefore, to study the effect of the $m v p$ deletions on the regulation of VRN2 we generated populations segregating for both genes simultaneously. The two $m v p$ deletions co-segregated with the non-flowering phenotype, but surprisingly, the lines homozygous for the $m v p$ mutations showed reduced transcript levels of both $V R N 2$ and $F T$ relative to the wild type. The VRN1 deletion is an unlikely cause of the down-regulation of VRN2 since VRN2 transcript levels are higher in the fall, before VRN1 is expressed, and are down-regulated by VRN1. Since both $V R N 2$ and $F T$ are regulated by light and photoperiod, their down-regulation in the $m v p$ mutants might be related to the deletion of the PHYC photoreceptor. However, alternative hypotheses including combinations of other genes deleted in the $m v p$ mutants cannot be ruled out. Until the specific gene(s) responsible for the down-regulation of VRN2 and $F T$ and the non-flowering phenotype are precisely identified, it is premature to use these results to postulate alternative flowering models.
\end{abstract}

Communicated by A. Schnittger.

A. Distelfeld · J. Dubcovsky ( $\square)$

Department of Plant Sciences, University of California,

One Shields Avenue, Davis, CA 95616, USA

e-mail: jdubcovsky@ucdavis.edu
Keywords Flowering · Wheat - Vernalization . Phytochrome $\cdot$ VRNI

\section{Introduction}

A precise regulation of flowering initiation is essential for the adaptation of wheat and barley varieties to different environments. Therefore, a better understanding of the regulatory gene network responsible for this developmental transition is required for an efficient manipulation of this trait in changing environments.

The recent discovery of two non-flowering mutants of cultivated diploid wheat (Triticum monococcum ssp. monococcum $\mathrm{L}$., $2 n=2 x=14$, genome $\mathrm{A}^{\mathrm{m}} \mathrm{A}^{\mathrm{m}}$ ) has provided a new tool to dissect the flowering regulatory network in the temperate cereals. These mutants, designated maintained vegetative phase ( $m v p)$, were generated by ion-beam radiation and remain indefinitely in the vegetative state independently of environmental stimuli (Shitsukawa et al. 2007).

On the contrary, wild type plants of the same species have the ability to initiate the reproductive phase in response to changes in photoperiod (day-length) and vernalization (long-term exposure to cold temperatures). In the temperate cereals, most of the natural variation in the response to photoperiod is concentrated in the $P P D 1$ gene (Turner et al. 2005; Beales et al. 2007; Wilhelm et al. 2009) whereas most of the natural variation in the response to vernalization occurs in the VRN1,VRN2 and VRN3 genes (Beales et al. 2007; Trevaskis et al. 2007; Distelfeld et al. 2009a). Throughout the text, a two letter prefix will be added to gene names to indicate the source species $(H v$, Hordeum vulgare; Ta, Triticum aestivum; Tm, T. monococcum). No prefix will be added when the effect is known to occur in all tested Triticeae species. 
VRN1 plays a central role in the regulation of flowering time and is responsible for most of the natural variation in vernalization requirement in wheat and barley. This gene encodes a MADS-box transcription factor with high similarity to the meristem identity genes APETALAl (API), CAULIFLOWER (CAL) and FRUITFULL (FRU) (Yan et al. 2003) which promote the transition of the vegetative shoot apical meristem (SAM) to the reproductive phase in Arabidopsis (Ferrandiz et al. 2000). The TmVRN2 locus includes two similar genes that encode proteins with a putative zinc finger and a CCT domain (TmZCCT1 and $T m Z C C T 2$ ) that acts as flowering repressors, with no clear homologues in Arabidopsis (Yan et al. 2004b). VRN3 encodes a RAF kinase inhibitor-like protein with high homology to Arabidopsis protein FLOWERING LOCUS T (FT) (Yan et al. 2006) that acts as a long-distance flowering signal (florigen).

The induction of the SAM to a reproductive stage is prevented in winter wheat and barley varieties by the presence of functional ZCCT1 or ZCCT2 genes (reviewed in Trevaskis et al. 2007; Distelfeld et al. 2009a) and a repressed state of the VRNI chromatin associated with high levels of histone 3 lysine 27 trimethylation (H3K27me3) (Oliver et al. 2009). Vernalization results in a stable reduction of $\mathrm{H} 3 \mathrm{~K} 27 \mathrm{me} 3$ and in parallel increases of other histone marks associated with an active chromatin state. The gradual increase in VRNI transcripts is associated with the repression of VRN2 (Loukoianov et al. 2005; Trevaskis et al. 2006) and the up-regulation of $F T$ (Distelfeld et al. 2009a). The up-regulation of FT requires long days, a process mediated by photoperiod genes PPDI and $C O$ (Beales et al. 2007; Turck et al. 2008). Long days are also required for the transcription of ZCCT1 and ZCCT2 (Dubcovsky et al. 2006; Trevaskis et al. 2006). Null or non-functional alleles for both ZCCT genes eliminate the vernalization requirement and result in spring growth habit (Yan et al. 2004b; Distelfeld et al. 2009b).

The strong epistatic interactions observed among the $V R N 1, V R N 2$ and VRN3 (FT) genes suggest that they are part of the same regulatory gene network. However, the existence of a feedback regulatory loop including these three genes has complicated the interpretation of different flowering experiments, and resulted in two alternative models for the regulation of flowering in the temperate cereals (Fig. 1). The first model, designated hereafter as the "VRN2-to-FT" model (Fig. 1a), suggests that VRN2 represses $F T$ and that $F T$ is a promoter of VRNI (Trevaskis et al. 2007; Distelfeld et al. 2009a). The alternative model, designated hereafter as the "FT-to-VRN2" model (Fig. 1b), suggests that VRNI promotes $F T$ and that $F T$ acts as a repressor of VRN2 (Shimada et al. 2009).

The VRN2-to-FT model (Fig. 1a), suggests that in the temperate cereals, FT acts in a similar way as the homeolo-

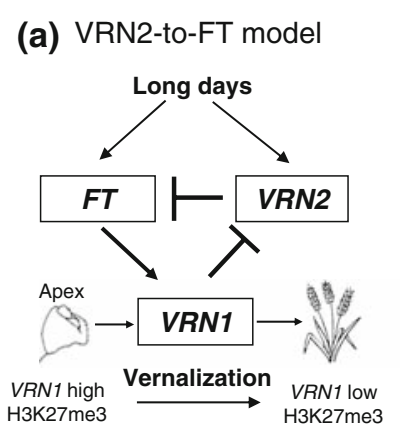

(b) FT-to-VRN2 model

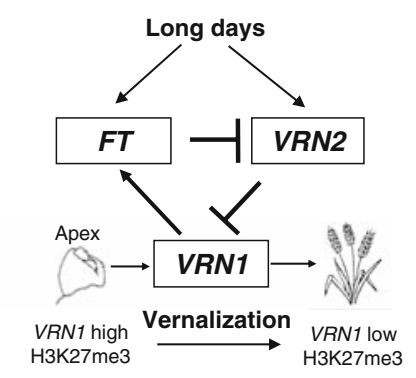

Fig. 1 General model of flowering in wheat. a In the model proposed by Distelfeld et al. (2009a, b) and Trevaskis et al. (2007), VRN2 is a repressor of $F T$, which promotes VRN1 under long days. VRN1 is upregulated by vernalization and is a repressor of $V R N 2$. b In the model proposed by Shimada et al. (2009), VRN1 is a promoter of $F T$ which operates as a repressor of $V R N 2$. In this model $V R N 2$ represses $V R N 1$

gous genes in Arabidopsis by promoting transcription of the meristem identity genes (Teper-Bamnolker and Samach 2005; Wigge et al. 2005). As in Arabidopsis, the wheat TaFT protein has been shown to interact with an FD-like protein (TaFDL2) that binds to the TmVRN1 promoter ( $\mathrm{Li}$ and Dubcovsky 2008). However, this model does not explain well the TmFT transcription profiles recently described in the non-flowering $m v p$ mutants (Shimada et al. 2009).

These non-flowering mutants carry induced deletions that encompass the complete TmVRN1 coding region and natural deletions of the TmVRN2 locus (Shitsukawa et al. 2007; Shimada et al. 2009). Recently, Shimada et al. (2009) showed that the $m v p$ mutants have very low transcript levels of TmFT in spite of the absence of the TmVRN2 repressor, an unexpected result based on the VRN2-to-FT model (Fig. 1a). To explain this result Shimada et al. (2009) proposed the alternative FT-to-VRN2 model (Fig. 1b), in which VRN1 promotes $F T$ transcription and its deletion explains the down-regulation of $F T$. However, this model contradicts the known interactions between Arabidopsis $F T$ and VRN1 homologues (AP1/CAL/FUL).

In order to clarify these conflicting models, we performed a more detailed molecular and genetic characterization of the $m v p$ mutants, and generated populations segregating simultaneously for the TmVRN1 and TmVRN2 deletions. An additional objective of the current study was to test the TmVRN2 response to vernalization in the absence of TmVRN1.

\section{Materials and methods}

Plant material and experimental design

The winter wheat accession G3116 (T. monococcum ssp. aegilopoides $\mathrm{L}$.) was used as the source of the functional TmVRN2 allele. This allele was introgressed into spring 
T. monococcum ssp. monococcum accession DV92 through six backcrosses which resulted in the winter line DV92:G3116-Vrn2 (Yan et al. 2004b). DV92:G3116-Vrn2 was crossed with the $\mathrm{M}_{2}-1921$ line of KU104-2 and $\mathrm{M}_{2}-1326$ line of KU104-1 (heterozygous for the $m v p-1$ and $m v p-2$ mutations, respectively). Hybrids from these two crosses were self-pollinated and two $\mathrm{F}_{2}$ populations, designates hereafter as pop- 1 and pop-2, respectively, were generated. Plants from these segregating populations were grown under long days (LD, $16 \mathrm{~h}$ light/8 h dark) and constant temperature $\left(20^{\circ} \mathrm{C}\right)$. The $\mathrm{F}_{2}$ plants were genotyped with TmVRN1 and TmVRN2 markers (see next section) and only those carrying at least one functional TmVRN2 copy were selected for the expression experiment. Among those, 12 non-flowering lines homozygous for the mvp TmVRN1 deletions and 12 with at least one copy of the wild type (WT) TmVRN1 gene were selected for the experiment. The first leaf samples for RNA extraction were collected when plants were 4 weeks old ( $4 \mathrm{~W}$ ) before starting the different temperature treatments. Half of the lines from each genotypic class were maintained at $20^{\circ} \mathrm{C}$ and the other half were transferred to a cold chamber $\left(5^{\circ} \mathrm{C}, \mathrm{LD}\right)$ for 6 weeks (vernalization treatment). After 6 weeks, a second set of leaf samples was collected for RNA extraction from the 10 -week-old $(10 \mathrm{~W})$ vernalized plants (before returning them to $20^{\circ} \mathrm{C}$ ) as well as from the non-vernalized controls.

\section{Genotyping}

\section{TmVRN1}

The $\mathrm{F}_{2}$ plants were genotyped for the presence or absence of TmVRN1 using primers 5'-AGCCACAAGAACCGGG ACTA-3' and 5'-CCCAAACTTTGCGGTGTATC-3' that amplified a 339-bp fragment from the TmVRN1 promoter region (BAC sequence AY188331). In addition, a codominant cleaved amplified polymorphism (CAP) marker was developed for the TRANSCRIPTIONAL ADAPTOR 2 (TmADA2) gene, which is adjacent to the deletion. This marker was used to differentiate plants homozygous and heterozygous for the presence of the TmVRN1 gene. TmADA2 primers 5'-GAAGATGCACTTGGAGAAGG-3' and 5'-GTCTCTTTGCATTGTACCCA-3' were used to amplify a 708-bp product from the parental lines DNAs. Digestion of the amplified product with restriction enzyme TaqI yielded two fragments of 30 and 678-bp in the $m v p$ lines (KU104-2 and KU104-1) and three fragments of 30, 185 and 493-bp in the DV92:G3116-Vrn2 line.

\section{TmVRN2}

Since TmVRN2 is deleted in both mvp mutants, we developed a codominant degenerate CAP marker for the adjacent
SNF2 gene (BAC sequence EF540321). Primers 5'-GTGG TTTGATTGGCATTCCT-3' and 5'-CAGACAACTGCG TCACCGTA-3' were used to amplify a 187-bp product from the parental lines DNAs. The underlined $G$ indicates a mutation introduced to generate an RsaI restriction site. Digestion of the amplified product with restriction enzyme $R s a$ I yielded three fragments of 19, 75 and 93-bp in the $m v p$ lines (KU104-2 and KU104-1) and two fragments of 75 and 112-bp in DV92:G3116-Vrn-2 line.

Primer pairs for the genomic copies of the T. monococcum AGAMOUS-LIKE GENE 1 (TmAGLG1), CYTOCHROME B5 (TmCYB5), CYSTEINE PROTEINASE (TmCYS), and PHYTOCHROME-C (TmPHYC) genes were described before (Yan et al. 2003).

Quantitative-PCR analysis

RNA was extracted using Spectrum Plant Total RNA Kit (Sigma-Aldrich, Saint Louis, MO, USA). First strand cDNA was synthesized from $1 \mu \mathrm{g}$ of total RNA with the QuantiTect Reverse Transcription Kit (Qiagen, Valencia, CA, USA). Quantitative-PCR was performed on an ABI PRISM 7000 SDS (Applied Biosystems, Foster City, CA, USA) using SYBR ${ }^{\circledR}$ GREEN and the previously published primer sets for the TmZCCT1 and TmZCCT2 (Distelfeld et al. 2009b) and TmFT (Yan et al. 2006). As an endogenous control, a fragment from the T. monococcum TRANSLATION ELONGATION FACTOR 1 (TEF1) gene was amplified with primers 5'-GCCCTCCTTGCTTTCACTCT-3' and 5'-AACGCGCCTTTGAGTACTTG-3' (91-bp, efficiency $99 \%$ ). The $2^{-\Delta \Delta C t}$ method (Livak and Schmittgen 2001) was used to normalize and calibrate transcript values relative to the endogenous control.

In addition, we developed primer sets for the RT-PCR studies of TmPHYC (5'-GGAGCAGAGGCAACTTTTTG-3' and $5^{\prime}$-ATTCCACCGTGTTCATCTCC-3', 119-bp), TmCYS (5'-GATCTTGGTCAGCCCTTCC-3' and 5'-GACCTCA CCGACGAGGAGT-3', 234-bp), and TmAGLG1 (5'-GCT GAAGGCAAGAGTTGAGG-3' and 5'-CTAGGGCCTG GAAGAAGTGC-3', 259-bp).

\section{Results}

Co-segregation of the mvp mutations and the non-flowering phenotype

The two $\mathrm{F}_{2}$ populations (pop-1 and pop-2) segregating for the $m v p-1$ and $m v p-2$ mutations showed a ratio of three flowering to one non-flowering plants (pop-1 49:10 $\chi^{2}$ $P=0.46$, pop-2 31:8 $\left.\chi^{2} P=0.75\right)$. All the plants that failed to flower were homozygous for the TmVRN1 deletion, whereas all the flowering plants carried at least one wild 
Table 1 The different genotypes and phenotypes of $p o p-1$ and $p o p-2$ derived from crosses between heterozygous lines for $m v p-1$ and $m v p-2$ with einkorn wheat accession DV92:G3116-Vrn2

\begin{tabular}{llll}
\hline Genotype & pop1 & pop2 & Phenotype \\
\hline Homozygous for $m v p-1$ deletion & 10 & 8 & Inability of the SAM to initiate reproductive phase \\
At least one copy of TmVRN1 and null allele of TmVRN2 & 10 & 9 & Spring growth habit, induced to flower without vernalization \\
At least one copy of TmVRN1 and functional TmVRN2 & 39 & 22 & Winter growth habit, induced to flower after 6 weeks vernalization \\
\hline
\end{tabular}

pop1, DV92:G3116-Vrn2 $\times \mathrm{M}_{2}$-1921 line of KU104-2

pop2, DV92:G3116-Vrn2 $\times \mathrm{M}_{2}-1326$ line of KU104-1

type TmVRN1 allele (Table 1). These results indicated that the non-flowering phenotype described before (Shitsukawa et al. 2007) is controlled by a single locus linked to the $m v p-1$ and $m v p-2$ deletions.

Among the flowering plants (carrying at least one copy of TmVRN1), those homozygous for the TmVRN2 deletion, flowered early and without vernalization ( $\sim 70$ days, spring growth habit) while those with at least one functional copy of TmVRN2 were induced to flower only after 6 weeks of vernalization (winter growth habit) (Table 1). This indicates that only TmVRN2 was segregating in these set of plants, and that both parental lines have the recessive Tmvrnl allele for winter growth habit. This was confirmed by sequencing of the TmVRN1 promoter region of the two mvp parental lines, which showed $100 \%$ identity to the TmVRN1 gene from winter T. monococcum accession G1777 (Yan et al. 2003).

Molecular characterization of the $m v p$ mutations

Shitsukawa et al. (2007) inferred that the deletion in the mvp-1 mutant was limited to a $19-\mathrm{kb}$ region including only the TmVRNl gene. This conclusion was based on two primer sets that successfully amplified PCR products from the $5^{\prime}(65,228-66,046)$ and $3^{\prime}(84,799-85,280)$ regions of the TmVRN1 gene using mvp-1 DNA. However, our analyses of these primers using the Triticeae repeat database (TREP-http://wheat.pw.usda.gov/ITMI/Repeats/blastrepeats3. $\mathrm{html}$ ) showed that the $65,228-66,046$ sequence was $100 \%$ identical to a CACTA DNA transposon (TREP1225) and the 84,799-85,280 sequence $100 \%$ identical to a Gypsy retrotransposon (TREP1240). Therefore, the PCR products obtained with these primers are likely to represent products from multiple repetitive loci and are not appropriate to infer the borders of the mvp- 1 and $m v p-2$ deletions.

The ion beams used to produce the $m v p$ mutants are known to induce large deletions that may include multiple genes (Hase et al. 1999). To test this possibility, we used specific primer pairs for six genes tightly linked to TmVRN1 (Yan et al. 2003) and tested their presence in the $m v p-1$ and $m v p-2$ mutants and parental lines (Fig. 2). The PCR products from genes TmCYB5 and TmADA2 were

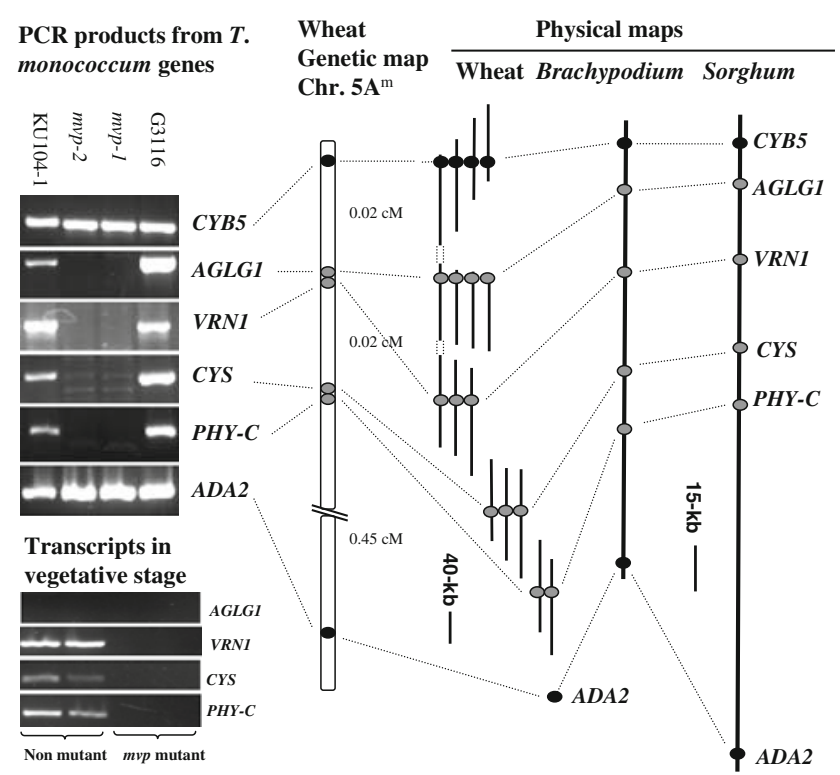

Fig. 2 Molecular characterization of the maintained vegetative phase ( $m v p$ ) deletions. DNAs from diploid wheat accessions G3116, mvp-1 mutant, $m v p-2$ mutant and KU104-1 (WT of the $m v p-1$ mutant) were tested for PCR amplification with primers for six genes from the TmVRN1 physical and genetic maps (Yan et al. 2003). Sequences from the colinear regions in sorghum (205-kb) and Brachypodium $(140-\mathrm{kb})$ are shown on the right. The primers for the TmCYB5 and TmADA2 genes (black circles) successfully amplified products from all DNA samples, whereas primers for TmAGLG1, TmVRN1, TmCYS and TmPHYC (gray circles) failed to amplify products from the genomic DNAs extracted from the $m v p-1$ and $m v p-2$ mutants. Transcripts of the TmPHYC, TmCYS, and TmVRN1 genes were detected in the leaves of the vernalized plants of non-mutant plants from pop- 1 and pop-2 but not in those homozygous for the mvp deletions. TmAGLG1 is known to be expressed in young spikes (Yan et al. 2003)

successfully amplified from all samples, but the primers for TmAGLG1, TmVRN1, TmCYS and TmPHYC, all failed to amplify any product in the $m v p-1$ and $m v p-2$ mutants. The same primers amplified fragments of the expected sizes in T. monococcum controls G3116 and KU104-1 (Fig. 2). The deleted genes (TmAGLG1, TmVRN1, TmCYS and TmPHYC) cover a $0.02 \mathrm{cM}$ region whereas the first genes present outside the deletion (TmCYB5 and TmADA2) are $0.5 \mathrm{cM}$ apart. This indicates that the $m v p-1$ and $m v p-2$ deletions encompass regions that are $0.02-0.5 \mathrm{cM}$ long. 
(a)

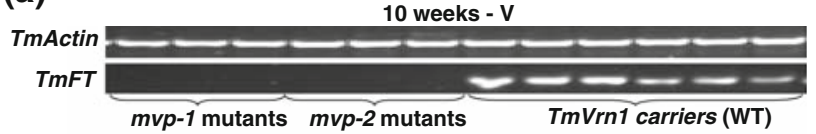

(b)

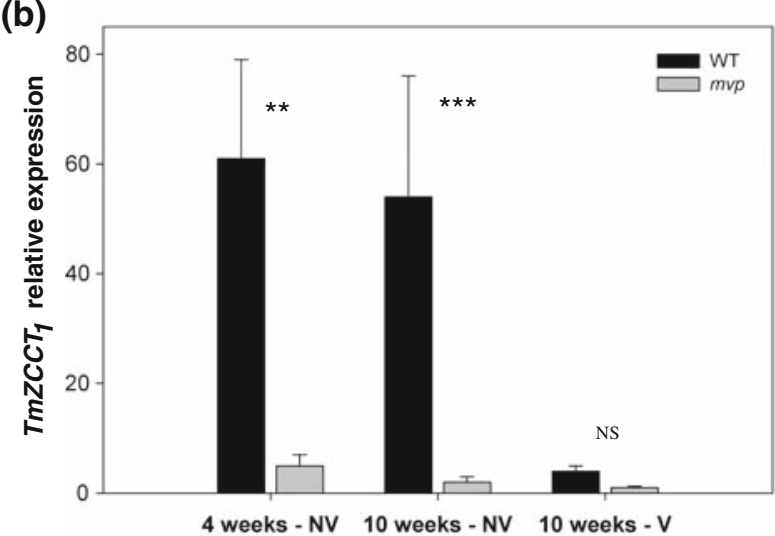

(c)

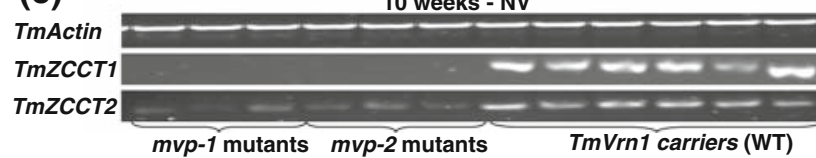

Fig. 3 Transcription profiles associated with the $m v p$ mutations. Transcript levels of TmVRN2 (TmZCCT1 and TmZCCT2) and TmFT were compared in leaf samples from $\mathrm{F}_{2}$ populations derived from a cross between heterozygous maintained vegetative phase mutants ( $m v p-1$ and $m v p$-2) and diploid winter lines (TmVRN2 carriers). a Semi Q-PCR analysis of plants after vernalization confirmed that the down-regulation of TmFT co-segregates with the $m v p$ deletions. b Expression of TmVRN2 (TmZCCT1) in $\mathrm{F}_{2}$ plants carrying at least one functional copy of TmVRN1 (WT, black bars) compared with $\mathrm{F}_{2}$ plants homozygous for the $m v p$ deletion ( $m v p$, gray bars). Comparisons were made at two time points (4 and 10-week-old plants), either with vernalization $(V)$ or without vernalization $(N V)$. No significant differences were observed between the two populations and therefore the data presented is an average of the two populations. $* *, * * *$ and NS indicate significance at $P \leq 0.01,0.001$ or non-significant effect, respectively, using Student's $t$ test. c Similar down-regulation was observed for the TmZCCT2 gene in 10 -week-old plants without vernalization treatment

Transcription profiles associated with the $m v p$ deletions

Shimada et al. (2009) showed that $T m F T$ transcripts are significantly reduced in the $m v p-1$ mutants relative to sister lines carrying functional TmVRNI alleles. This result was extended here to the $m v p-2$ mutants and to the segregating lines in pop1 and pop2 (Table 1; Fig. 3a), confirming that the down-regulation of TmFT is the result of the $m v p$ deletion in the TmVRN1 region and not of mutations in unlinked genes.

The effect of the TmVRN1 deletion on TmZCCTI and TmZCCT2 transcript levels could not be studied in the original $m v p$ mutants because both lines are homozygous for the natural TmVRN2 deletions (Shimada et al. 2009). The study of the TmZCCT1 and TmZCCT2 transcript levels in plants from pop- 1 and pop- 2 carrying these genes and segregating for the $m v p$ deletions, yielded a surprising result. The quantitative PCR results showed that TmZCCTI and TmZCCT2 transcript levels were significantly lower $(P<0.001)$ in the $m v p$ mutants than in the wild type sister plants even in the absence of vernalization (Fig. 3b). The low TmVRN2 transcript levels in the mvp mutants were similar to those observed in the wild type winter plants after vernalization (Fig. 3b). The differences in TmZCCT1 and TmZCCT2 transcript levels were linked to the $m v p$ deletions in both populations (Fig. 3c). In summary, the $m v p$ deletions were associated with the down-regulation of both TmVRN2 and TmFT genes compared to their wild type sister lines.

We also analyzed the transcript levels of the other genes present in the mvp deletions in pop1 and pop 2 plants with and without the $m v p$ deletions. As expected, no expression of the deleted genes TmAGLG1, TmVRN1, TmCYS and TmPHYC was detected in the mvp mutants (Fig. 2). The $F_{2}$ plants with at least one copy without the $m v p$ deletions showed abundant transcript levels for TmCYS and TmPHYC (Fig. 2). TmVRN1 was expressed at high levels only in the vernalized wild type plants. TmAGLG1 transcripts were not detected in any of the leaf samples supporting the results from Yan et al. (2003) that concluded that this gene is expressed in the spike after flowering initiation.

\section{Regulation of TmVRN2 by light}

The unexpected down-regulation of TmFT and TmVRN2 observed in the $m v p$ mutants parallels the down-regulation of these two genes under short days (Dubcovsky et al. 2006; Yan et al. 2006). In addition, in wheat plants grown under long days, TaFT shows higher expression in the light than in the dark (Shimada et al. 2009).

To study the response of TmVRN2 transcript levels to light, two different experiments were performed. In the first experiment, 8-week-old non-vernalized plants of T. monococcum accession G3116 (winter growth habit) previously grown under long day conditions were moved into the dark after $6 \mathrm{~h}$ of light (at noon). Analysis of TmZCCT1 and TmZCCT2 expression showed a rapid decrease in transcripts level during the $5 \mathrm{~h}$ of the experiment (Fig. 4a, b). A mirror image of this result was obtained in the second experiment, in which plants were moved to the light after being kept for $24 \mathrm{~h}$ in dark. In this experiment, TmZCCT1 and TmZCCT2 transcript levels increased rapidly during the $4 \mathrm{~h}$ of exposure to light (Fig. 4c, d). 
Fig. 4 Expression of $V R N 2$ (TmZCCT1 and TmZCCT2) under different light conditions in einkorn wheat. Expression of TmZCCT1 (a) and TmZCCT2 (b) when plants were transferred from light to dark. Expression of $T m Z C C T 1$ (c) and TmZCCT2 (d) when plants were transferred from dark to normal light
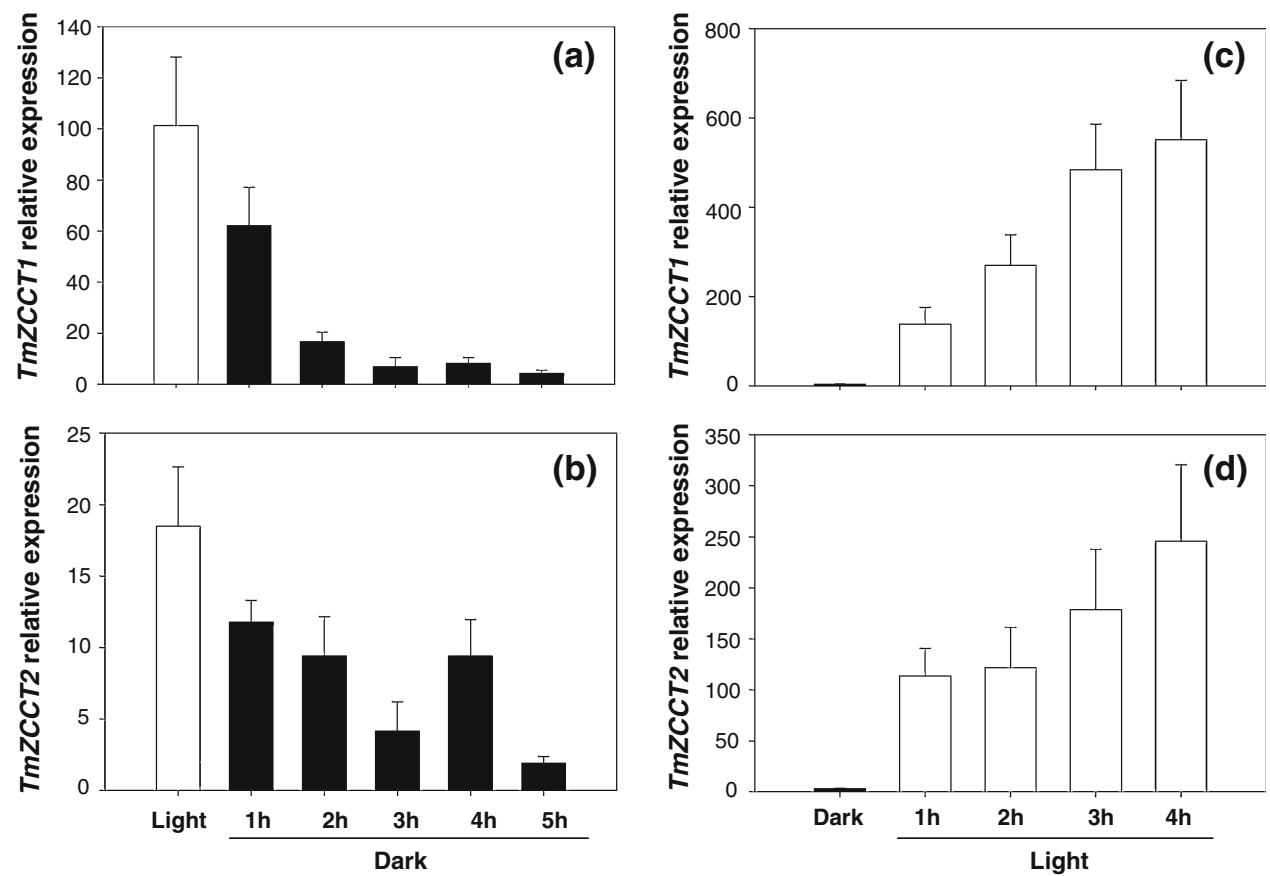

\section{Discussion}

Conflicting flowering models

The cloning and characterization of the vernalization genes VRN1 (Trevaskis et al. 2003; Yan et al. 2003) and VRN2 (Yan et al. 2004b) was the basis for the first flowering model for temperate cereals. In this initial model VRN2 was proposed to act as a transcriptional repressor (direct or indirect) of $V R N 1$, which is responsible for promoting the transition of the SAM to the reproductive stage. Temperate cereals with a spring growth habit have either deletions or non-functional mutations in the coding region of the VRN2 repressor (Yan et al. 2004b; Dubcovsky et al. 2005; von Zitzewitz et al. 2005; Distelfeld et al. 2009b) or deletions in regulatory regions of the VRN1 gene (Yan et al. 2004a; Fu et al. 2005; Hemming et al. 2009). This simple model explains the dominant nature of the Vrn2 alleles for winter growth habit (one functional copy is sufficient to repress flowering) and the dominant nature of the Vrnl mutations for spring growth habit (the deletion of a regulatory region in a single copy results in unregulated promotion of flowering). This model also explains well the known epistatic interactions between these two genes (Takahashi and Yasuda 1971; Tranquilli and Dubcovsky 2000).

Subsequent studies using isogenic lines of hexaploid wheat carrying different combinations of dominant and recessive TaVRN-A1, TaVRN-B1 and TaVRN-D1 alleles suggested that VRN1 is able to repress VRN2 (directly or indirectly) (Loukoianov et al. 2005). In these isogenic lines, the dominant TaVrnl alleles are expressed earlier in plant development and are followed later by the down-regulation of TaVRN2 and the up-regulation of the recessive Tavrn1 alleles in the other genomes suggesting the existence of a feedback regulatory loop (Loukoianov et al. 2005). The down-regulation of $H v V R N 2$ after the induction of $H v V R N 1$ was also observed in barley (Trevaskis et al. 2006).

The discovery that VRN2 was down-regulated by short days (Dubcovsky et al. 2006; Trevaskis et al. 2006) and that VRNI was up-regulated by vernalization under short days both in diploid wheat and barley (Dubcovsky et al. 2006; Trevaskis et al. 2006; Fu et al. 2007), led to the suggestion that VRN1 was the gene responsible for the perception of the vernalization signal (Trevaskis et al. 2006), which was followed later by other authors (Distelfeld et al. 2009a). Recently, this hypothesis received strong experimental support from the discovery that vernalization is associated with changes in the levels of several histone modifications in the chromatin of VRN1 but not in the chromatin of VRN2 or FT (Oliver et al. 2009).

The cloning of VRN3 (Yan et al. 2003) added an additional element to the initial flowering models. This gene is orthologous to rice $H d 3 a$ and Arabidopsis FT, which are both known to encode for proteins (florigen) that travel through the phloem to the SAM and promote flowering (Corbesier et al. 2007; Tamaki et al. 2007). In Arabidopsis, it was demonstrated that the FT protein interacts with FD in the SAM and that this complex binds to the AP1 promoter (Wigge et al. 2005). A similar mechanism has been postulated in wheat where TaVRN3 $(\sim \mathrm{FT})$ interacts with TaFDL2 ( FD), which has the ability to bind the wheat TmVRN1 promoter (Li and Dubcovsky 2008). In the 
temperate cereals, $F T$ integrates the signals from the photoperiod pathway through its interactions with $P P D 1$ and $C O$ (Turner et al. 2005) and the vernalization pathway through its interactions with VRN2 (Yan et al. 2006; Hemming et al. 2008; Distelfeld et al. 2009a). Based on the previous results, $F T$ was placed in the flowering model as an intermediate step in the VRN2 repression of VRN1 (Fig. 1a, VRN2-to-FT model). According to this model the high levels of $V R N 2$ during the long days of fall prevent the expression of $F T$, which limits the up-regulation of VRN1. The up-regulation of $V R N 1$ during the winter results in the down-regulation of $V R N 2$, the release of $F T$ from its repression and, under long days, the $F T$ up-regulation of $V R N I$ transcripts beyond the threshold required to initiate flowering (Fig. 1a).

The model presented in Fig. 1a explains well all the known epistatic interactions among these genes and their expression profiles in different genotypes and environmental conditions. However, this model fails to explain the down-regulation of TmFT observed in the mvp mutants lacking TmVRN1 (Shimada et al. 2009). To explain this last result, Shimada et al. (2009) proposed that TmVRN1 acts as a transcriptional promoter of $T m F T$, and that the absence of TmVRN1 in the mvp mutants is the cause of the down-regulation of TmFT (Fig. 1b). Although the FT-to-VRN2 model recognizes the existence of a feedback regulatory loop among the VRN1, VRN2 and VRN3 genes it reverts the order of the interactions among these genes relative to the VRN2-to-FT model (Fig. 1). Both models can be used to explain most of the experimental results. For example, the up-regulation of VRN1 transcripts in FT over-expressing transgenic plants (Shimada et al. 2009) can be explained by the FT-to-VRN2 model as an indirect effect mediated by the down-regulation of VRN2; or by the VRN2-to FT model as a direct up-regulation of $V R N 1$ by $V R N 3$ (Fig. 1). The existence of the feedback regulatory loop complicates the interpretation of most of the experiments, since modifications on any of these genes results in direct and indirect effects on the other genes in the loop. The absence of TmVRNI in the $m v p$ mutants interrupts this feedback loop and, therefore, provides a valuable tool to validate these models.

TmVRN2 expression profiles in the $m v p$ mutants conflict with both flowering models

In the FT-to-VRN2 model (Fig. 1b) the deletion of the $V R N 1$ gene is associated with the down-regulation of $F T$, and therefore it is expected to result in the up-regulation of $V R N 2$ (this model considers $F T$ as a repressor of $V R N 2$ ). To test this prediction, the TmZCCT1 and TmZCCT2 transcript levels were studied in the presence and absence of the TmVRNI gene.
Surprisingly, in the pop1 and pop2 segregating populations the plants homozygous for the $m v p$ deletions showed very low $T m Z C C T 1$ and TmZCCT2 transcript levels, even though the plants were not vernalized and were grown under long days (Fig. 3b). Under the same conditions, the plants carrying functional TmVRN1 and TmVRN2 genes, showed TmZCCT1 transcript levels more than sixfold higher than the plants homozygous for the $m v p$ deletions. These plants had winter growth habit and flowered more than 1 month later than the plants homozygous for the TmVRN2 deletion.

There was a perfect co-segregation between the homozygous $m v p$ deletions and the TmVRN2 down-regulation in the populations segregating for both $m v p$ mutations (Fig. 3c) suggesting that this down-regulation is a result of the $m v p$ deletions (or a gene linked to it). This result contradicts the expected increase of VRN2 transcripts in the absence of VRN1 predicted by both models (Fig. 1a, b) and therefore, it indicates that either both models are incorrect or that the observed changes in TmVRN2 (and TmFT) expression in the lines carrying the $m v p$ deletions is the result of other genes deleted in these mutants.

It is interesting to point out here that a simultaneous down-regulation of VRN2 and $F T$ transcript levels is observed when photoperiod sensitive wheat and barley plants are grown under short days (Dubcovsky et al. 2006; Hemming et al. 2008), or when plants grown under long days are in the dark (Shimada et al. 2009). Based on these observations we hypothesized that the gene affecting the $F T$ and VRN2 transcription profile might be associated with light or photoperiod and searched for those genes in the deleted region of the $m v p$ mutants.

Genes deleted in the $m v p$ mutants of einkorn wheat

In this study, we demonstrated that the deletions present in both the $m v p-1$ and $m v p-2$ mutants are larger than thought before (Shitsukawa et al. 2007) and include several genes in addition to TmVRN1 (Fig. 2). Homozygocity for these large deletions co-segregated with the lack of flowering and the down-regulation of TmVRN2 and TmFT, which suggests that these phenotypes are caused by one or several genes present within the $m v p$ deletions. Therefore, a description of the genes present within these deletions is relevant to understand the potential candidate genes for these different effects.

The $0.45 \mathrm{cM}$ region between TmPHYC and TmADA2 (Fig. 2) has not been sequenced yet in wheat and therefore, the putative wheat genes present in this region were inferred from the colinear region in Sorghum bicolor and Brachypodium distachyon (98 and 52-kb, respectively, Fig. 2). This Sorghum region has thirteen annotated genes but nine of them are just predicted hypothetical genes and 
their predicted proteins show no similarity to any plant proteins (Sb01g007855/860/874/876/890/910-Sb01g007940). The other four genes include a member of metal ion binding family (Sb01 g007870), a gene similar to EMBRYONIC FLOWER 2 (Sb01g007878), a gene similar to the isoleucyl tRNA synthetase (Sb01g007890) and an oligopeptide transporter (Sb01g007900). The Brachypodium colinear region includes only six genes, four that are just predicted hypothetical genes, a gene similar to an oligopeptide transporter (Bradilg08420, 58\% similar at the protein level to the sorghum orthologue) and a gene similar to the proteinase inhibitor I9 (Bradilg08450). None of these genes seem to be related to the photoperiod or light sensing pathways.

The distal part $(0.04 \mathrm{cM})$ of the $m v p$ deletion was sequenced in T. monococcum (Yan et al. 2003) and three genes, in addition to TmVRN1, were identified; TmAGLG1, TmCYS and TmPHYC. TmAGLG1 is a MADS-box gene related to the Arabidopsis $A G L 2$ subgroup and is expressed only in developing spikes (Yan et al. 2003) suggesting that it is an unlikely candidate to regulate flower initiation in the SAM. The CYSTEINE PROTEINASE gene (TmCYS) belongs to a large family of proteins that are involved with a variety of proteolytic functions in higher plants, particularly those associated with the processing and degradation of seed storage proteins and fruit ripening. They are also induced in response to stresses such as wounding, cold, drought and in programmed cell death and senescence processes (Prins et al. 2008). TmCYS transcript levels were abundant in the leaves of the non-mvp mutants in our two segregating populations (Fig. 2).

Finally, the TmPHYC gene which is also deleted in the $m v p$ mutants, belongs to a family of red/far-red photoreceptors that also includes the PHYA and $P H Y B$ genes (Furuya 1993; Devos et al. 2005). Rice PHYC mutants exhibit a partial loss of sensitivity to continuous red light and a moderate early flowering under long-day photoperiod (Takano et al. 2005). The phyC phyA double mutant shows a dramatic early flowering in rice, suggesting that these genes are implicated in the regulation of flowering repression (Takano et al. 2005). In Arabidopsis, the PHYC gene also contributes to variation in flowering time (Balasubramanian et al. 2006), and the PHYA and PHYB are known to be involved in the posttranscriptional regulation of $\mathrm{CO}$ and therefore, contribute to the regulation of the photoperiod pathway (Valverde et al. 2004). The clear association of the PHYC gene with the regulation of flowering initiation and the known role of the phytochromes in general on light signaling suggests that the deletion of TmPHYC in the $m v p$ mutants may have an effects on the regulation of TmVRN2 and/or TmFT. We confirmed here that the TmPHYC transcripts are abundant in the leaves of T. monococcum (Fig. 2) where TmVRN2 and TmFT are also expressed.
Since several genes are deleted in both $m v p$ mutants it is currently not possible to determine which of them is responsible for the lack of flowering and the transcriptional down-regulation of the VRN2 and FT genes. It is possible that different genes within this deletion are responsible for different combinations of the observed phenotypes. Our preferred hypothesis is that the VRN1 deletion is responsible for the non-flowering phenotype whereas the PHYC deletion is responsible for the down-regulation of VRN2 and $F T$ in non-vernalized plants grown under long day conditions.

The putative role of the meristem identity gene VRNI on the non-flowering phenotype is supported by a similar phenotype in Arabidopsis triple mutant apl cau ful, which lacks the three VRN1 homologues simultaneously (Ferrandiz et al. 2000). However, the $m v p$ phenotype seems to be more severe than the Arabidopsis triple mutant, in which limited flowering was observed under heat stress conditions (Ferrandiz et al. 2000). We currently do not know if this stronger flowering repression is the result of the combination of the TmVRN1 and TmPHYC deletions in the $m v p$ mutants or simply a stronger effect of the TmVRNI deletion in flowering initiation in T. monococcum.

The putative role of the $P H Y C$ gene on the down-regulation of the VRN2 and $F T$ is supported by the rapid response of these genes to light signals. When light conditions change from dark to light or from light to dark, a rapid change in VRN2 (Fig. 4) or FT (Shimada et al. 2009) transcript levels is observed. In addition, all current flowering models propose an effect of photoperiod on the regulation of VRN2 and FT (Fig. 1) (Trevaskis et al. 2007; Distelfeld et al. 2009a). Therefore, if PHYC is part of this photoperiodic regulation, no additional changes would be required in the current flowering models to explain the down-regulation of VRN2 and $F T$ in the mvp mutants. In contrast, if the down-regulation of $F T$ and VRN2 were the result of the deletion of VRNI, then both flowering models (Fig. 1a, b) would require substantial modifications because both predict an up-regulation of VRN2 in the absence of VRN1.

To provide a more conclusive answer to these questions and to determine whether single gene effects can recapitulate the individual $m v p$ phenotypes, we have initiated the production of wheat mutants for the VRNI and PHYC genes using our wheat TILLING populations (Uauy et al. 2009). Until the specific gene(s) responsible for the down-regulation of VRN2 and $F T$ and the non-flowering phenotype are precisely identified, it is premature to use these results to postulate alternative flowering models.

Acknowledgments We would like to thank Dr. Koji Morai for kindly providing us with the heterozygous lines for the $m v p-1$ and $m v p-2$ mutations. This project was supported by the National Research Initiative grants number 2007-35301-17737 and 2007-35301-18188 from the USDA National Institute of Food and Agriculture. Dr. Assaf 
Distelfeld was supported by a Vaadia-BARD Postdoctoral Fellowship Award No. FI-386-06. The authors thank Cindy Miguita and Mei-Yee Lau for excellent technical assistance.

Open Access This article is distributed under the terms of the Creative Commons Attribution Noncommercial License which permits any noncommercial use, distribution, and reproduction in any medium, provided the original author(s) and source are credited.

\section{References}

Balasubramanian S, Sureshkumar S, Agrawal M, Michael TP, Wessinger C, Maloof JN, Clark R, Warthmann N, Chory J, Weigel D (2006) The PHYTOCHROME C photoreceptor gene mediates natural variation in flowering and growth responses of Arabidopsis thaliana. Nat Genet 38:711-715

Beales J, Turner A, Griffiths S, Snape JW, Laurie DA (2007) A Pseudo-Response Regulator is misexpressed in the photoperiod insensitive Ppd-Dla mutant of wheat (Triticum aestivum L.). Theor Appl Genet 115:721-733

Corbesier L, Vincent C, Jang SH, Fornara F, Fan QZ, Searle I, Giakountis A, Farrona S, Gissot L, Turnbull C, Coupland G (2007) FT protein movement contributes to long-distance signaling in floral induction of Arabidopsis. Science 316:1030-1033

Devos KM, Beales J, Ogihara Y, Doust AN (2005) Comparative sequence analysis of the Phytochrome $C$ gene and its upstream region in allohexaploid wheat reveals new data on the evolution of its three constituent genomes. Plant Mol Biol 58:625-641

Distelfeld A, Li C, Dubcovsky J (2009a) Regulation of flowering in temperate cereals. Curr Opin Plant Biol 12:178-184

Distelfeld A, Tranquilli G, Li C, Yan L, Dubcovsky J (2009b) Genetic and molecular characterization of the VRN2 loci in tetraploid wheat. Plant Physiol 149:245-257

Dubcovsky J, Chen C, Yan L (2005) Molecular characterization of the allelic variation at the $V R N-H 2$ vernalization locus in barley. Mol Breed 15:395-407

Dubcovsky J, Loukoianov A, Fu D, Valarik M, Sanchez A, Yan L (2006) Effect of photoperiod on the regulation of wheat vernalization genes VRN1 and VRN2. Plant Mol Biol 60:469-480

Ferrandiz C, Gu Q, Martienssen R, Yanofsky MF (2000) Redundant regulation of meristem identity and plant architecture by FRUITFULL, APETALA1 and CAULIFLOWER. Development 127:725-734

Fu D, Szücs P, Yan L, Helguera M, Skinner J, Hayes P, Dubcovsky J (2005) Large deletions in the first intron of the $V R N-1$ vernalization gene are associated with spring growth habit in barley and polyploid wheat. Mol Genet Genomics 273:54-65

Fu D, Uauy C, Blechl A, Dubcovsky J (2007) RNA interference for wheat functional gene analysis. Transgenic Res 16:689-701

Furuya M (1993) Phytochromes-their molecular species, gene families, and functions. Annu Rev Plant Physiol Plant Mol Biol 44:617-645

Hase Y, Shimono K, Inoue M, Tanaka A, Watanabe H (1999) Biological effects of ion beams in Nicotiana tabacum L. Radiat Environ Biophys 38:111-115

Hemming MN, Peacock WJ, Dennis ES, Trevaskis B (2008) Low-temperature and daylength cues are integrated to regulate FLOWERING LOCUS T in barley. Plant Physiol 147:355-366

Hemming MN, Fieg S, Peacock WJ, Dennis ES, Trevaskis B (2009) Regions associated with repression of the barley (Hordeum vulg are) VERNALIZATION1 gene are not required for cold induction. Mol Genet Genomics 282:107-117

Li C, Dubcovsky J (2008) Wheat FT protein regulates VRN1 transcription through interactions with FDL2. Plant J 55:543-554
Livak KJ, Schmittgen TD (2001) Analysis of relative gene expression data using real-time quantitative PCR and the $2^{-\Delta \Delta C \mathrm{t}}$ method. Methods 25:402-408

Loukoianov A, Yan L, Blechl A, Sanchez A, Dubcovsky J (2005) Regulation of $V R N-1$ vernalization genes in normal and transgenic polyploid wheat. Plant Physiol 138:2364-2373

Oliver SN, Finnegan EJ, Dennis ES, Peacock WJ, Trevaskis B (2009) Vernalization-induced flowering in cereals is associated with changes in histone methylation at the VERNALIZATION1 gene. Proc Natl Acad Sci USA 106:8386-8391

Prins A, Van Heerden PDR, Olmos E, Kunert KJ, Foyer CH (2008) Cysteine proteinases regulate chloroplast protein content and composition in tobacco leaves: a model for dynamic interactions with ribulose-1, 5-bisphosphate carboxylase/oxygenase (Rubisco) vesicular bodies. J Exp Bot 59:1935-1950

Shimada S, Ogawa T, Kitagawa S, Suzuki T, Ikari C, Shitsukawa N, Abe T, Kawahigashi H, Kikuchi R, Handa H, Murai K (2009) A genetic network of flowering time genes in wheat leaves, in which an APETALA1/FRUITFULL-like gene, VRN1, is upstream of FLOWERING LOCUS T. Plant J 58:668-681

Shitsukawa N, Ikari C, Shimada S, Kitagawa S, Sakamoto K, Saito H, Ryuto H, Fukunishi N, Abe T, Takumi S, Nasuda S, Murai K (2007) The einkorn wheat (Triticum monococcum) mutant, maintained vegetative phase, is caused by a deletion in the VRN1 gene. Genes Genet Syst 82:167-170

Takahashi R, Yasuda S (1971) Genetics of earliness and growth habit in barley. In: Nilan RA (ed) Proceedings of the 2nd international barley genetics symposium. Washington State University Press, Washington, pp 388-408

Takano M, Inagaki N, Xie XZ, Yuzurihara N, Hihara F, Ishizuka T, Yano M, Nishimura M, Miyao A, Hirochika H, Shinomura T (2005) Distinct and cooperative functions of phytochromes A, B, and $\mathrm{C}$ in the control of deetiolation and flowering in rice. Plant Cell 17:3311-3325

Tamaki S, Matsuo S, Wong HL, Yokoi S, Shimamoto K (2007) Hd3a protein is a mobile flowering signal in rice. Science 316:10331036

Teper-Bamnolker P, Samach A (2005) The flowering integrator FT regulates SEPALLATA3 and FRUITFULL accumulation in Arabidopsis leaves. Plant Cell 17:2661-2675

Tranquilli G, Dubcovsky J (2000) Epistatic interactions between vernalization genes $V r n-A^{m} 1$ and $V r n-A^{m} 2$ in diploid wheat. J Hered 91:304-306

Trevaskis B, Bagnall DJ, Ellis MH, Peacock WJ, Dennis ES (2003) MADS box genes control vernalization-induced flowering in cereals. Proc Natl Acad Sci USA 100:13099-13104

Trevaskis B, Hemming MN, Peacock WJ, Dennis ES (2006) HvVRN2 responds to daylength, whereas $H v V R N 1$ is regulated by vernalization and developmental status. Plant Physiol 140:1397-1405

Trevaskis B, Hemming MN, Dennis ES, Peacock WJ (2007) The molecular basis of vernalization-induced flowering in cereals. Trends Plant Sci 12:352-357

Turck F, Fornara F, Coupland G (2008) Regulation and identity of florigen: FLOWERING LOCUS T moves center stage. Annu Rev Plant Biol 59:573-594

Turner A, Beales J, Faure S, Dunford RP, Laurie DA (2005) The pseudo-response regulator $\mathrm{Ppd}-\mathrm{H} \mathrm{l}$ provides adaptation to photoperiod in barley. Science 310:1031-1034

Uauy C, Paraiso F, Colasuonno P, Tran RK, Tsai H, Berardi S, Comai L, Dubcovsky J (2009) A modified TILLING approach to detect induced mutations in tetraploid and hexaploid wheat. BMC Plant Biol 9:115-129

Valverde F, Mouradov A, Soppe W, Ravenscroft D, Samach A, Coupland G (2004) Photoreceptor regulation of CONSTANS protein in photoperiodic flowering. Science 303:1003-1006 
von Zitzewitz J, Szűcs P, Dubcovsky J, Yan L, Francia E, Pecchioni N, Casas A, Chen THH, Hayes PM, Skinner JS (2005) Molecular and structural characterization of barley vernalization genes. Plant Mol Biol 59:449-467

Wigge PA, Kim MC, Jaeger KE, Busch W, Schmid M, Lohmann JU, Weigel D (2005) Integration of spatial and temporal information during floral induction in Arabidopsis. Science 309:1056-1059

Wilhelm EP, Turner AS, Laurie DA (2009) Photoperiod insensitive Ppd-Ala mutations in tetraploid wheat (Triticum durum Desf.). Theor Appl Genet 118:285-294

Yan L, Loukoianov A, Tranquilli G, Helguera M, Fahima T, Dubcovsky J (2003) Positional cloning of wheat vernalization gene VRN1. Proc Natl Acad Sci USA 100:6263-6268
Yan L, Helguera M, Kato K, Fukuyama S, Sherman J, Dubcovsky J (2004a) Allelic variation at the $V R N-1$ promoter region in polyploid wheat. Theor Appl Genet 109:1677-1686

Yan L, Loukoianov A, Blechl A, Tranquilli G, Ramakrishna W, SanMiguel P, Bennetzen JL, Echenique V, Dubcovsky J (2004b) The wheat $V R N 2$ gene is a flowering repressor down-regulated by vernalization. Science 303:1640-1644

Yan L, Fu D, Li C, Blechl A, Tranquilli G, Bonafede M, Sanchez A, Valarik M, Dubcovsky J (2006) The wheat and barley vernalization gene VRN3 is an orthologue of FT. Proc Natl Acad Sci USA 103:19581-19586 